\title{
Limited value of the resting electrocardiogram in assessing patients with recent onset chest pain: lessons from a chest pain clinic
}

\author{
Cardiology \\ Harefield Hospital, \\ Harefield, Middlesex \\ M Norell \\ D Lythall \\ G Coghlan \\ A Cheng \\ S Kushwaha \\ J Swan \\ C Ilsley \\ A Mitchell \\ Correspondence to: \\ Dr Michael Norell, \\ Cardiology Department, \\ Royal Free Hospital, Pond \\ Street, Hampstead, London \\ NW3 2QG \\ Accepted for publication \\ 12 September 1991
}

\author{
M Norell, D Lythall, G Coghlan, A Cheng, S Kushwaha, J Swan, C Ilsley, A Mitchell
}

\begin{abstract}
Objective-To evaluate a clinic set up specifically to assess patients with recent onset chest pain, particularly those presenting with a normal resting electrocardiogram.
\end{abstract}

Design-Retrospective review of case notes.

Setting-Cardiac department of a tertiary referral cardiothoracic centre.

Patients-250 consecutive patients with recent onset chest pain seen within 24 hours of general practitioner referral.

Outcome measures-Clinical diagnosis and management.

Results- $40 \%$ of patients were seen within seven days of the onset of symptoms. Twenty seven per cent had non-cardiac symptoms and could be discharged while $60 \%$ were considered to have cardiac pain. Sixty six patients (26\%) were admitted directly from the clinic and 48 of these underwent coronary angiography within three weeks. Seventy patients $(28 \%)$ have so far undergone intervention (angioplasty or coronary artery surgery), 22 within one month of presentation. One hundred and nine patients $(44 \%)$ presented with a normal resting electrocardiogram, 21 of whom were considered to have unstable angina. Forty one of these patients were investigated of whom 37 were found to have significant coronary disease and 26 have undergone intervention.

Conclusions-This experience highlights the inadequacy of a routine electrocardiogram reporting service in patients with recent onset of chest pain. An alternative facility offering immediate and complete cardiac assessment produced patient benefit with early diagnosis and intervention. Investigation of these patients, however, accounted for $5 \%$ of cardiac catheterisation laboratory throughput; this was a significant additional and unscheduled workload.

The value of the resting electrocardiogram in patients with suspected acute infarction is clear. ${ }^{1}$ Its role in the management of patients with recent onset chest pain which may have a cardiac cause, however, is poorly defined. Patients with long-standing symptoms may be referred to a cardiological outpatient depart- ment but those with shorter histories need an early diagnosis. Waiting lists for outpatient appointments can result in diagnostic delay in patients with possible unstable coronary disease or unnecessarily prolong anxiety in patients with non-cardiac symptoms. Referral to an accident and emergency department may be viewed as unjustified in patients without acute symptoms and junior casualty department staff may not be sufficiently experienced to make an adequate assessment. ${ }^{2}$ These patients are frequently referred to their local hospital for an electrocardiogram, the report of which takes no account of the clinical presentation. Thus this investigation may be misleading or falsely reassuring.

Alternatively, these patients may be offered a complete assessment in a dedicated daily clinic. We established such a facility to assess specifically patients with possible cardiac pain of recent onset and here we report our experience of the first 250 patients seen in this chest pain clinic.

\section{Patients and methods}

A chest clinic was established in October 1988 at Harefield Hospital, a regional cardiothoracic centre, which also serves general practitioners responsible for a local population of 150000 . Newly referred outpatients are usually seen within eight weeks and up to 20 such patients are seen weekly. A single cardiac catheterisation laboratory investigates 2200 patients annually and 250 angioplasties and 650 coronary bypass surgical procedures are performed annually. The current wait for diagnostic cardiac catheterisation is between four and six months.

A circular was sent to 120 local general practitioners to inform them of a daily facility offering full assessment of patients presenting with chest pain of recent onset. General practitioners were encouraged to telephone the on-call cardiology registrar to discuss the case before sending the patient to the clinic which was available between 2 and 4 pm every week day.

All patients were seen on the day of referral and assessed by a cardiology registrar or senior registrar who recorded the clinical diagnosis based on the history, physical examination, and resting electrocardiogram.

Unstable angina was defined as any deterioration in the pattern of previously stable symptoms, rest pain, or only exertional symptoms if they had been present for less than two 
Figure 1 Duration of symptoms in the study group (" $90+"$ indicates patients who had symptoms for more than three months).

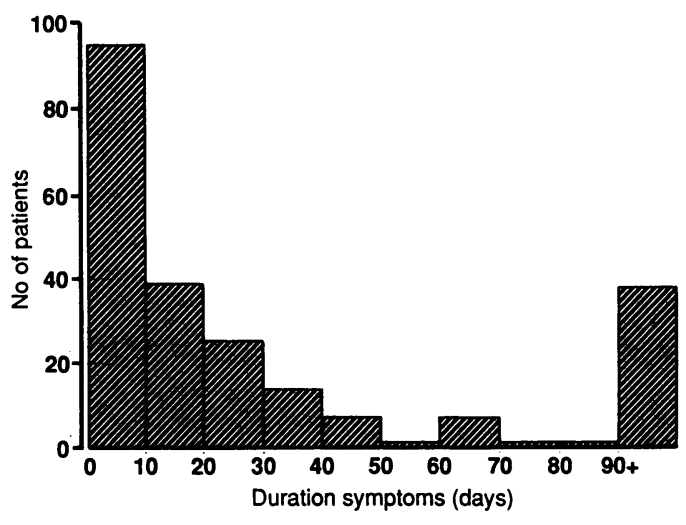

weeks. Significant coronary disease was defined angiographically as a $50 \%$ or greater reduction in luminal diameter of at least one major coronary vessel.

Further investigations-for example, exercise electrocardiography-or hospital admission, were arranged if appropriate and the diagnosis, action taken, and outcome were recorded and reviewed.

\section{Results}

After the establishment of the chest pain clinic in October 1988, 250 patients (176 men and 74 women, mean age 58 years (range 25-84 years)) were assessed over the next 18 months. All patients were seen within 24 hours of referral by their general practitioner.

\section{DURATION OF SYMPTOMS}

The mean duration of symptoms precipitating referral was 27 days (range 1-90 days) (fig 1) One hundred and eighty seven patients (75\%) presented within 30 days of the onset of symptoms and $100(40 \%)$ were assessed within seven days.

Thirty eight patients (15\%) had had symptoms for more than three months with no discernible recent deterioration (fig 1 , column $90+$ days).

\section{CLINICAL DIAGNOSIS}

Sixty nine patients $(27.5 \%)$ were considered to have non-cardiac pain (fig 2). In a further 29 patients $(11.5 \%)$ the cause of chest pain was not clear after clinical assessment and further investigations were arranged. In 146 cases $(58.5 \%)$ the diagnosis was of angina and in half of these the patient's symptoms were considered to represent unstable angina, despite a normal resting electrocardiogram in 21 patients. Six patients $(2.5 \%)$ presented with myocardial infarction.

Seventy three patients had exercise testing on the day of presentation and in most cases this aided the clinical diagnosis.

\section{PATIENT OUTCOME}

Sixty three patients considered to have noncardiac pain $(25.2 \%)$ were discharged from the clinic without further follow up. In a further 59 cases $(23.6 \%)$ arrangements were made for outpatient review with the result of further investigations-for example exercise electrocardiography-or to monitor the effect of changes in medical treatment.

Over half the patients $(128$ cases, $51 \cdot 2 \%)$ had coronary arteriography, with significant coronary disease being found in 114. Sixty two of these were investigated within 30 days of presentation and 35 were studied within seven days. Sixty six patients $(26 \%)$ required admission directly to the ward for stabilisation on medical treatment. Of these 48 underwent coronary arteriography within 21 days.

Thirty one patients $(12.4 \%)$ had coronary artery bypass surgery and $39(15.6 \%)$ coronary angioplasty. These interventions were performed within 30 days of presentation in 22 and within seven days of presentation in nine.
Figure 2 Clinical diagnosis in 250 patients.

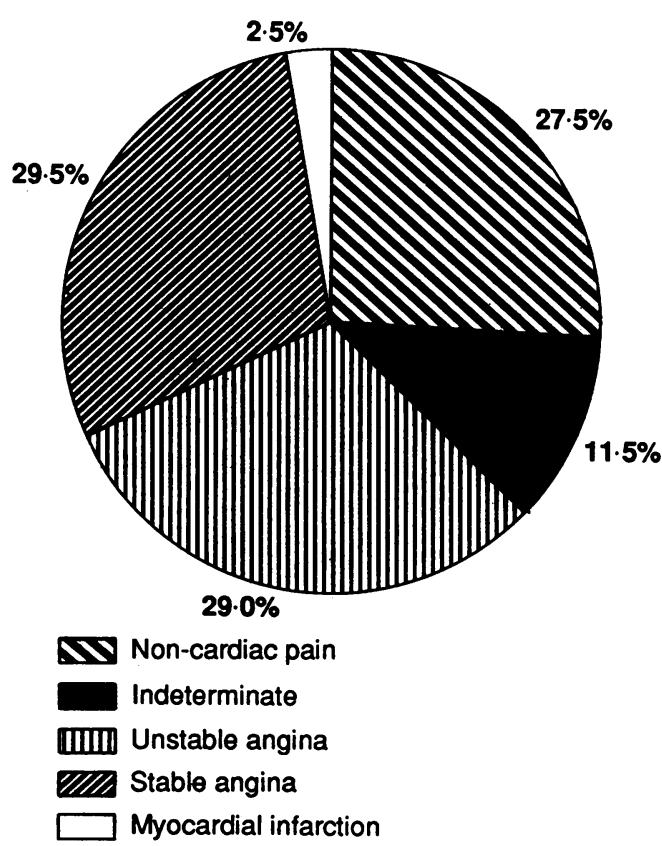

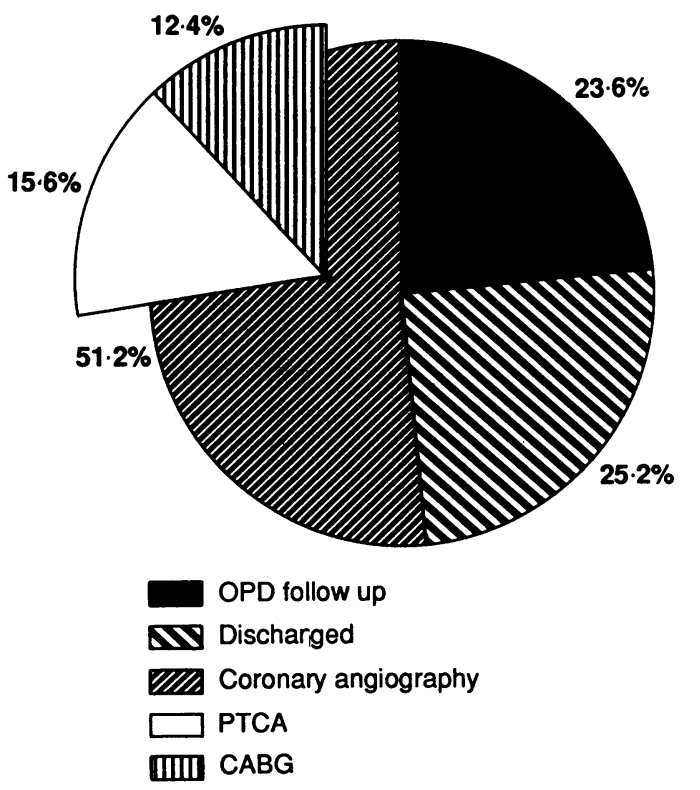

Figure 3 Outcome in 250 patients. CABG, coronary artery surgery; $P T C A$, percutaneous transluminal coronary angioplasty; OPD, outpatients department. 
Figure 4 (A) Clinical diagnosis in 109 patients presenting with a normal electrocardiogram. (B) Outcome in 109 patients presenting with a normal presenting with a normal footnote to figure 3 for abbreviations.
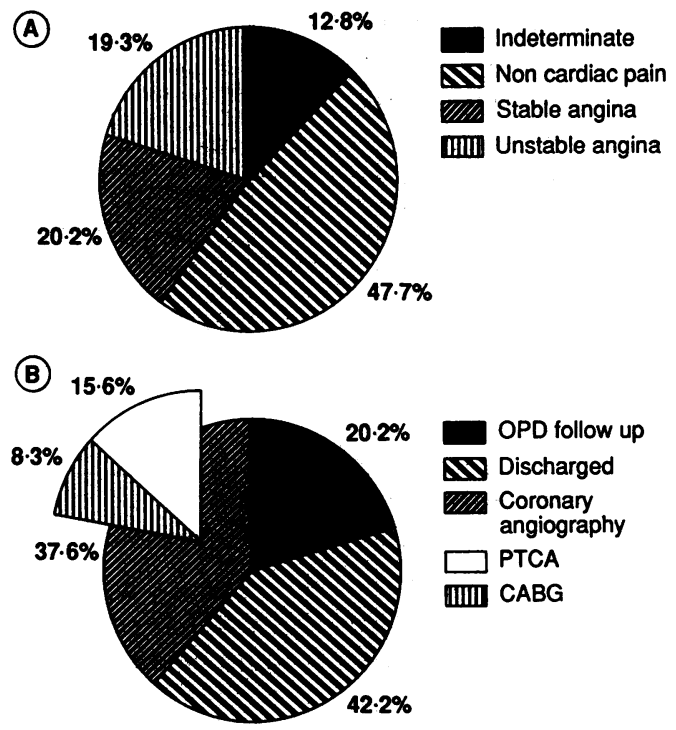

\section{DIAGNOSIS AND OUTCOME IN PATIENTS WITH} NORMAL ELECTROCARDIOGRAMS

One hundred and nine (44\%) patients presented with a normal resting electrocardiogram. In almost half of this group ( 52 cases, $47 \cdot 7 \%$ ) the diagnosis was of non-cardiac pain while in a further 14 cases $(12.8 \%)$ the cause of chest pain was undetermined (fig 4).

Outpatient follow up was arranged for 22 patients $(20 \cdot 2 \%)$ and 46 patients $(42 \cdot 2 \%)$ were discharged without further review being planned.

However, in 43 cases (39.5\%) the diagnosis was of cardiac pain and despite a normal resting electrocardiogram half of these patients were considered to have unstable angina. Forty one patients $(37.6 \%)$ in this group had coronary arteriography, which showed significant coronary disease in 37 . Coronary angioplasty has been undertaken in 17 patients $(15.6 \%)$ and coronary artery surgery in nine cases $(8 \cdot 3 \%)$.

\section{Discussion}

This initial experience of a chest pain clinic suggests that patients with symptoms of recent onset may benefit from early cardiac assessment. Though these data are uncontrolled it is likely that early investigation and intervention in some cases was advantageous. A quarter of our patients had undergone cardiac catheterisation as a definitive diagnostic procedure within one month of presentation. Similarly $10 \%$ had undergone either coronary angioplasty or coronary artery surgery within the same period. This is considerably shorter than our routine waiting time for cardiac catheterisation and intervention.

The most important finding in this study was the outcome of patients with normal electrocardiograms. In our study $20 \%$ of these were considered to have unstable angina and they constituted almost a third of all patients with that diagnosis. In the absence of electrocardiographic changes the diagnosis in these circumstances must be a clinical one but it is supported by the requirement for either coron- ary artery surgery or angioplasty in a quarter of our patients with normal electrocardiograms.

This experience highlights the potential dangers associated with the finding of a normal resting electrocardiogram in patients with suspected unstable angina. In a practice serving 2500 patients, a general practitioner may see up to 100 patients with chest pain per year. ${ }^{3}$ Our results show that in up to one third of these patients a normal resting electrocardiogram may be seriously misleading and, therefore, the value of a routine electrocardiography reporting service must be questionable. Some general practitioners have access to their own electrocardiograph ${ }^{4}$ which may relieve some of the load from hospital departments. However, even their correct interpretation of the electrocardiogram would not avoid the reassurance of a falsely normal result. For the same reasons referral to an accident and emergency department would not confer further advantage. ${ }^{2}$

Patients referred for either coronary arteriography or angioplasty as a result of attending our chest pain clinic accounted for almost $5 \%$ of the annual throughput of our cardiac catheterisation laboratory. In the absence of a chest pain clinic it is likely that, eventually, these patients would have required investigation and treatment, thus constituting the same annual load on resources. However, accident and emergency department or outpatient referral in these cases would undoubtedly have led to delay in investigation and treatment. Furthermore these procedures represent an unplanned workload which has important implications in terms of the availability of hospital beds and impact on an already full cardiac catheterisation list.

A diagnosis of acute myocardial infarction was made in only six cases despite referral of patients specifically with suspected cardiac symptoms. However, the population assessed in our clinic was selected after discussion with the general practitioner in each case. Because patients were to be seen in an outpatient department we believed that in those cases where the diagnosis of acute myocardial infarction was likely the general practitioner would be better advised to send the patient to the nearest accident and emergency department. There, full resuscitative facilities would be immediately available on arrival if required.

A quarter of the patients seen had noncardiac pain. We did not regard this finding as a waste of clinic resources because this provided a valuable service to a sizeable number of patients who required reassurance. A wait for outpatient referral could have engendered unnecessary anxiety; early assessment with exercise electrocardiography, if appropriate, meant that outpatient attendance was then unnecessary.

\section{CLINICAL IMPLICATIONS}

In patients with chest pain of recent onset, a normal resting electrocardiogram cannot exclude unstable angina or, in some cases, acute infarction. ${ }^{5}$ The value of a routine electrocardiogram reporting service is therefore questionable. 
A chest pain clinic run by specifically trained staff offers rapid assessment of patients with suspected cardiac symptoms of recent onset and allows early investigation and intervention if appropriate. However, such a facility has important consequences for hospital resources in terms of bed availability and the workload of the cardiac catheterisation laboratory.
1 Timmis A. Early diagnosis of acute myocardial infarction. BMJ 1990:301.941-2.

2 McCallion WA, Templeton PA, McKinney LA, Higginson JDS. Interpretation of electrocardiograms in the accident and emergency department [abstract]. Br Heart J 1990, and ex.

$3 \mathrm{Mead} M$, Paterson H. Tutorials in general practice. London: Pitman, 1983

4 Davies A. Electrocardiographs in general practice. $B M J$ 1989;299:408-9.

5 Yusuf S, Pearson M, Sterry H, et al. The entry ECG in the early diagnosis and prognostic stratification of patients with suspected acute myocardial infarction. Eur Heart J 1984;5:716-26.

\section{Comment}

We all can cite cases where a patient complaining of chest pain had a normal electrocardiogram in Accident and Emergency and was discharged with tragic consequences. Norell et al found that 43 of 109 patients with recent onset of chest pain and a normal electrocardiogram subsequently had an unequivocal clinical diagnosis of cardiac pain. In nearly all of them (41) this diagnosis was confirmed at coronary angiography. Subsequently 26nearly a quarter - of the original group with a normal electrocardiogram had coronary angioplasty or coronary artery surgery. This shows that we must not rely on a normal electrocardiogram to exclude important coronary disease in patients with recent onset of chest pain. Ideally, if resources permit, these patients should be assessed later by exercise testing.

Norell and his colleagues have given us a useful measure of the true size of this potentially dangerous problem. The message is not new to cardiologists but now that we see the size of the problem we must alert general practitioners, general physicians, and junior doctors in casualty departments and remind them that the electrocardiogram and patient should always be examined together.

PETER MILLS

The Royal London Hospital,

Whitechapel Wondon E1 IBB 